\title{
Dominio de habilidades del pensamiento computacional en los estudiantes del Instituto Tecnológico Superior Sucre de Quito - Ecuador
}

\author{
Mastery of computational thinking skills in the students of the Sucre \\ Superior Technological Institute of Quito - Ecuador
}

\author{
Hamilton Omar Pérez Narváez \\ Universidad Central del Ecuador (Ecuador) \\ hperez@uce.edu.ec \\ Alex Álvarez-Zurita \\ Universidad Central del Ecuador (Ecuador) \\ amalvarez@uce.edu.ec \\ Carlos Roberto Guevara Herrera \\ Profesor Instituto Tecnológico Superior "Sucre" \\ cquevara@tecnologicosucre.edu.ec
}

Recibido: 29/09/2019

Aceptado: 9/12/2019

Publicado: 26/12/2019

\section{RESUMEN}

El pensamiento computacional y las habilidades relacionadas con él son de interés tanto para el campo educativo, así como para el campo profesional, pues la habilidad de resolución de problemas apoyada en la informática se convierte en un elemento necesario para las personas que viven en la sociedad actual. La investigación tuvo como propósito dos situaciones: diagnosticar el nivel de desarrollo del pensamiento computacional en los estudiantes del Instituto Tecnológico Superior "Sucre", así como establecer las relaciones existentes entre las habilidades seleccionadas del mismo como son orientación espacial, toma de decisiones, empleo de bucles y elaboración de funciones o subrutinas para la resolución de problemas. Con esta finalidad se aplicó un instrumento validado que consta de 32 problemas para resolver en un ambiente gráfico conformadas de la siguiente manera: 4 preguntas acerca de direcciones, 8 sobre bucles definidos e indefinidos, 12 sobre toma de decisiones, 8 acerca de funciones y subrutinas. Las conclusiones más importantes establecen fortalezas de los estudiantes en relación con el empleo de algoritmos de dirección y empleos de bucles para la resolución de problemas algorítmicos, así como hallaron dificultades en el empleo de toma de decisiones y uso de subrutinas. Además, establece que existe relación entre el desarrollo que tengan en la habilidad toma decisiones y otras habilidades como empleo de bucles de repetición o elaboración de subrutinas, que se expresa en la dificultad de los estudiantes a tomar una decisión razonada y lógica al momento de resolver un problema.

\section{PALABRAS CLAVE}

Pensamiento lógico; pensamiento computacional; habilidades de información; evaluación. 


\section{ABSTRACT}

Computational thinking and its skills are of interest for the educational field, as well as for the professional field, since the problem-solving ability supported by computer science becomes a necessary element for people living in the actual society. The aim of the investigation was two situations: to diagnose the level of computational thinking development in the students of the Instituto Tecnológico Superior "Sucre", as well as to establish connections between the selected skills, such as spatial orientation, decision making, employment loops and elaboration of functions or subroutines for problem solving. For this purpose, a validated instrument was applied; it consists of 32 problems to solve in a graphic environment formed as follows: 4 questions about directions, 8 about defined and indefinite loops, 12 about conditional ones, 8 about functions and subroutines. The most important conclusions establish students' strengths in relation to the use of management algorithms and loop jobs, similarly, difficulties in the use of conditions and use of subroutines were found. Besides, it establishes that there is a relationship between the development they have in the decision-making ability and other skills such as the use of repetition loops or the elaboration of subroutines, which is expressed in the students' difficulty in making a reasoned and logical decision at the time of solve a problem.

\section{KEYWORDS}

Logical thinking, computational thinking, information skills, evaluation

\section{CITA RECOMENDADA}

Pérez, H.O. (2019). Dominio de habilidades del pensamiento computacional en los estudiantes del Instituto Tecnológico Superior Sucre de Quito - Ecuador. RIITE. Revista Interuniversitaria de Investigación en Tecnología Educativa, 7, 48-60. Doi: http://dx.doi.org/10.6018/riite.394221

\section{Principales aportaciones del artículo y futuras líneas de investigación:}

- Establecimiento de la línea base acerca de las habilidades del pensamiento computacional en el Instituto Tecnológico Superior Sucre

- Relacionar las habilidades del pensamiento computacional evaluadas en la solución de problemas

- Aplicación del instrumento en otras instituciones de educación superior estableciendo el nivel de desarrollo del pensamiento computacional con la intención de reingresar las ciencias computacionales a la malla curricular de la educación general básica y bachillerato en el Ecuador.

- Diseño de metodologías de enseñanza y aprendizaje para el desarrollo de habilidades del pensamiento computacional.

\section{INTRODUCCIÓN}

La sociedad actual ha considerado la adquisición de nuevas habilidades a las tradicionalmente requeridas, tanto en la vida profesional como en las actividades cotidianas. Por ello se han considerado aquellas que tienen relación con el uso de la tecnología informática como importantes para esta época.

Al respecto Scott (2015) menciona que esta integración de nuevos conocimientos se debe a situaciones como:

La mundialización, las nuevas tecnologías, las migraciones, la competencia internacional, la evolución de los mercados y los desafíos medioambientales y políticos transnacionales son todos ellos factores que rigen la adquisición de las competencias y los conocimientos que las y los estudiantes necesitan para sobrevivir y salir airosos en el siglo XXI. (p. 2).

Dentro de estas habilidades necesarias, para los profesionales al igual que para el resto de personas, el pensamiento computacional va adquiriendo una importancia en los últimos años, de 
manera que se han elaborado propuestas para su desarrollo desde las etapas iniciales de educación formal como en el caso del K-12 del Reino Unido, donde se recomienda por parte de Royal Society od Education y la Royal Academy of Engineering (2012) "Se debe implementar un plan de acción, con vistas a permitir que todos los jóvenes tengan la oportunidad de estudiar Tecnología de la Información y Ciencias de la Computación" (p. 8) con la intención de desarrollar en los estudiantes conocimientos informáticos aplicables a la industria y los negocios que los países desarrollados requieren.

En los Estados Unidos, la CSTA e ISTE, organizaciones relacionadas con la enseñanza y desarrollo de las Ciencias de la Computación, respectivamente, coinciden en la importancia del tema, señalan que al ser llamadas a integrarse dentro del proyecto de incluir el pensamiento computacional en la formación de los estudiantes estadounidenses aceptaron "el compromiso con el firme principio de que todos los estudiantes debían demostrar competencia en las habilidades básicas del pensamiento computacional, en el momento de graduarse o finalizar la educación escolar" (CSTA-ISTE, 2011, p.4).

El término pensamiento computacional, tiene su génesis en el documento de Wing (2006) publicado por el ACM donde expresa: "El pensamiento computacional es una habilidad fundamental para todos, no solo para los informáticos. A leer, escribir y realizar operaciones aritméticas, deberíamos agregar el pensamiento computacional pensando en la capacidad analítica de cada niño" (p. 33).

Posteriormente, Wing (2008) explica mejor la relación del PC con otras formas de pensamiento de la siguiente manera:

El pensamiento computacional es una clase de pensamiento analítico. Comparte con pensamiento matemático en las maneras generales en qué podríamos aproximarnos a solucionar un problema. Comparte con el pensamiento de ingeniería en las maneras generales en qué podríamos acercarnos a diseñar y evaluar un sistema grande, complejo que opera dentro de las condiciones del mundo real. Comparte con el pensamiento científico en las maneras generales en qué nos podríamos acercar a la computación comprensiva, inteligencia, la mente y comportamiento humano. (p. 3717).

Del análisis documental acerca del tema, se puede considerar como un término en construcción, que propone encontrar convergencias entre distintas formas de pensamiento, empleando la tecnología de manera crítica y creativa, con la intención de resolver problemáticas en diferentes disciplinas del conocimiento y de la sociedad.

Durante estos años, también otros países han desarrollado propuestas para incluirlo en sus planes de estudio, de manera que niños y jóvenes estén en capacidad de resolver problemas apoyados en las posibilidades que brinda los sistemas informáticos.

En América Latina, países como Chile y Colombia han apostado por adaptaciones curriculares que permitan su inclusión. En el caso chileno a nivel escolar, Hitschfeld, Pérez y Simmonds (2015) consideran que esta decisión permitiría dar un salto de país consumidor de tecnología a productor de la misma. Colombia lo ha incluido en la educación media conjuntamente con el modelo de formación STEM con el propósito que sus estudiantes se incluyan en las innovaciones educativas que están presentes a nivel mundial.

En el caso de Ecuador, según reporta Pérez (2017) "las experiencias educativas en el pensamiento computacional son escasas y aisladas, principalmente relacionadas con el uso de Scratch" (p. 21) y no se han establecido mediante investigaciones el desarrollo que tienen los estudiantes de los diferentes niveles educativos: inicial, básico y bachillerato en las habilidades que conforman el PC. Tampoco existen evidencian de propuestas a nivel superior que integren este tipo de pensamiento en la formación profesional que desarrollan las instituciones educativas en los niveles de grado y psogrado.

Actualmente, se han elaborado varios instrumentos con la finalidad de evaluar el desarrollo del pensamiento computacional que han sido validados, por lo que establecer cómo se han desarrollado estas habilidades en instituciones de educación superior para comparar con otros estudios a nivel nacional e internacional es una actividad importante y necesaria que posteriormente permitirá diseñar propuestas curriculares que aporten a complementar la formación del estudiante. 
A lo señalado anteriormente, se suma un cambio de política educativa del gobierno actual en Ecuador, que con el diseño de la Agenda Educativa Digital una colaboración entre los Ministerios de Educación y de Telecomunicaciones (Ministerio de Educación del Ecuador, 2017), manifiesta la intención de incluir en la formación de los estudiantes de educación básica y bachillerato, una asignatura denominada Ciencias de la Computación a la que consideran "es una materia que promueve la integración del área de pensamiento computacional en el currículo nacional, desarrollando habilidades de pensamiento claves como el de razonamiento lógico, la modelización, la abstracción y la resolución de problemas" (p. 26).

Sin coincidir con esta propuesta el organismo rector de la educación superior ecuatoriana, SENESCYT, plantea eliminar la Informática de la malla curricular que deben trabajar los institutos tecnológicos superiores, sin que exista un estudio que establezca realmente cuanto se han desarrollado las habilidades relacionadas con el pensamiento computacional en los futuros tecnólogos del país.

Por todo lo mencionado, se determinó la necesidad de realizar la investigación acerca de las habilidades relacionadas con el pensamiento computacional que poseen los estudiantes del Instituto Tecnológico Superior "Sucre" de la ciudad de Quito, encargado de la formación de jóvenes que desean obtener un título de tecnólogo en las especialidades de: electricidad, electrónica y electromecánica industrial,

Para la investigación se establecieron, entre los elementos relacionados con el PC, las siguientes dimensiones: orientación espacial, toma de decisiones, bucles o repetición de procesos y subrutinas o creación de funciones.

La orientación espacial, a decir de Suárez, Maiz y Meza (2010) "consiste en formar un modelo mental del mundo en tres dimensiones. Es la inteligencia que tienen los marineros, los ingenieros, los cirujanos, los escultores, los arquitectos, o los decoradores" (p.87). En el resto de personas nos permite ubicarnos, así como ubicar objetos, apreciando las distancias o la manera de dirigirnos a ellos.

La toma de decisiones corresponde a una situación cotidiana, puesto que diariamente realizamos infinidad de elecciones de acuerdo con lo que nos proponemos alcanzar.

Al respecto, Kahneman (2011) menciona:

Tomar decisiones es como hablar en prosa, la gente lo hace todo el tiempo, lo sepa o no. Por eso no resulta sorprendente que la lógica de la toma de decisiones la compartan muchas disciplinas, desde las matemáticas y la estadística, pasando por la economía y la ciencia política, hasta la sociología y la psicología. (p. 437).

Entonces, de vital importancia desarrollar en las personas la toma de decisiones, basada principalmente, en su razonamiento por sobre otros factores.

La repetición de procesos facilita replicar acciones de manera automática logrando eficiencia en las acciones. Esta habilidad en la programación, permite repetir sentencias de forma automática o condicional, de acuerdo a lo escrito por el programador, asegurando la ejecución de las líneas que estén dentro del mismo.

Las subrutinas, define Llopis (1998) como:

Uno de los métodos fundamentales para resolver un problema es dividirlo en problemas más pequeños, llamados subproblemas, pudiendo éstos, a su vez, ser subdivididos repetidamente en problemas más pequeños hasta llegar a obtener subprogramas lo suficientemente reducidos como para que resuelvan una única tarea y sean, por tanto, programados de forma más sencilla. (p. 91).

Esta habilidad del pensamiento computacional es similar al proceso denominado análisis que es aplicable a otras disciplinas del conocimiento, así como a la vida diaria. 


\section{MÉTODO}

\subsection{Objetivos}

Los objetivos que orientaron la investigación fueron:

- Diagnosticar el nivel de desarrollo en habilidades del pensamiento computacional en los estudiantes del Instituto Tecnológico Superior "Sucre" empleando un instrumento validado internacionalmente.

- Establecer las relaciones entre habilidades algorítmicas del pensamiento computacional para la solución de problemas en los estudiantes del Instituto Tecnológico Superior "Sucre".

En concordancia con los objetivos se plantearon las siguientes preguntas de investigación:

1. ¿Cuál es el nivel de dominio en las habilidades del pensamiento computacional en los estudiantes del Instituto Tecnológico Superior "Sucre"?

2. ¿Existe relación entre el dominio de la orientación espacial y la toma de decisiones en la resolución de problemas?

3. ¿Existe relación entre el dominio sobre la toma de decisiones y el empleo de bucles de repetición en la resolución de problemas?

4. ¿Existe relación entre el dominio de toma de decisiones y el empleo de funciones o subrutinas en la resolución de problemas?

\subsection{Participantes}

Los involucrados en la investigación corresponden a 336 estudiantes del Instituto Tecnológico Superior "Sucre" de la ciudad de Quito de las especialidades: electricidad, electrónica y electromecánica industrial durante el semestre 2017-2018, matriculados desde primero a sexto, que colaboraron en el llenado del instrumento.

La Tabla 1 resume el número de estudiantes por cursos:

Tabla 1. Población general divida por cursos

\begin{tabular}{llrr} 
& Número de estudiantes & Porcentaje \\
\hline Curso & 91 & 27,1 \\
2 & 127 & 37,8 \\
3 & 7 & 2,1 \\
4 & 57 & 17,0 \\
5 & 36 & 10,7 \\
6 & 18 & 5,4 \\
\cline { 2 - 4 } & Total & 336 & 100,0
\end{tabular}

Fuente: Secretaría Instituto Tecnológico Superior "Sucre" 2017. Elaboración: Investigadores

Ateniendo al género la población estudiantil, fueron 307 de género masculino mientras que 29 correspondieron al género femenino. La Figura 1 a continuación muestra esta distribución. 


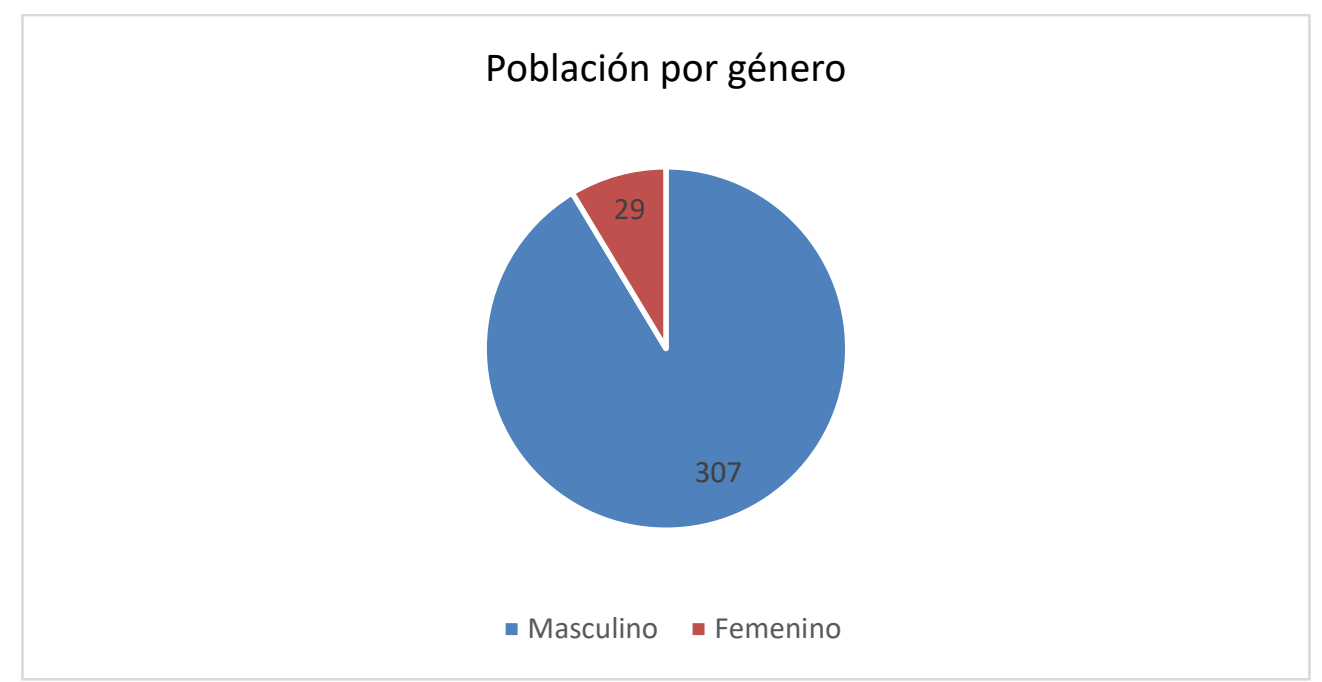

Figura 1. Género del estudiantado participante. Fuente: Elaboración propia.

\subsection{Enfoque de investigación}

Se seleccionó para el estudio un enfoque cuantitativo, guardando relación con el tipo de instrumento que elaboró para el cuestionario que permitió recopilar información de los involucrados.

Por el alcance la investigación fue a nivel descriptivo y correlacional con apoyo de investigación documental.

\subsection{Procedimiento}

En resumen, se procedió en la investigación de la siguiente forma:

\section{Fase 1. Preparación de la investigación}

Establecido el problema de investigación y el grupo de colaboradores, se sostuvo una conversación previa con las autoridades, donde se solicitó el permiso para realizar la investigación en la institución. Además se aseguró la participación de los estudiantes y el apoyo de los docentes.

También, se mantuvo contactos con los investigadores españoles que elaboraron el cuestionario denominado Computational Thinking Test 1 (Román, Pérez y Moreno, 2016), para evaluar el pensamiento computacional. Contando con el permiso de los autores se procedió a adaptarlo, principalmente en lo correspondiente al lenguaje debido a las expresiones idiomática propias del cada país y el tipo de ejercicios propuestos.

\section{Fase 2. Recolección de información}

Luego, se implementó mediante la plataforma de Google Drive, un sitio para que los estudiantes llenen los cuestionarios de forma que los resultados fueran alojados automáticamente en una hoja de cálculo. Los estudiantes que estaban empezando un nuevo ciclo académico en los diferentes cursos de la especialidad fueron encuestados, solicitando su participación.

\section{Fase 3. Análisis e interpretación}

Para el procesamiento de la información se empleó el software estadístico SPSS, obteniendo medidas de tendencia central y frecuencias, al igual que gráficos de entre los cuales se seleccionó los más representativos organizando por aciertos y desaciertos.

En lo que se refiere a establecer la relación entre habilidades del pensamiento computacional, se seleccionó el coeficiente de correlación de Pearson, debido que en durante el análisis se optó por considerarlas como variables cuantitativas. 
Finalmente, los resultados permitieron llegar a conclusiones que se resumen en el artículo.

\subsection{Instrumentos}

El instrumento empleado es un cuestionario compuesto por datos personales, género y semestre de estudio, posteriormente posee tres ejemplos de cómo trabajar en la selección de opciones y finalmente presenta al estudiante 35 problemas en ambiente gráfico con cuatro tipos de solución que el estudiante puede escoger, incluyendo el ítem denominado no conoce.

Se compone de dos secciones: la primera corresponde a datos informativos y la segunda se compone de problemas algorítmicos que emplean distintas sentencias relacionadas al pensamiento computacional, conformadas de la siguiente manera: 4 preguntas acerca de orientación espacial, 8 sobre bucles definidos e indefinidos, 12 sobre toma de decisiones 0 condicionales y 11 problemas acerca de funciones y subrutinas.

Como se mencionó con anterioridad, la autoría del instrumento corresponde a Román, Pérez y Moreno (2016) quienes lo describen de la siguiente manera "Computational Thinking Test 1 (CTt) es un instrumento de opción múltiple [...] que se administran en línea (a través de dispositivos electrónicos móviles o no móviles) en un tiempo máximo de 45 minutos" (p. 3).

Se puede señalar que el instrumento reúne las características apropiadas para evaluar el pensamiento computacional, ya que, al realizar el análisis de fiabilidad mediante el Alfa de Cronbach, alcanza un valor de 0,743; de acuerdo con García-Bellido, González y Jornet (2015) es considerado como aceptable tomando en cuenta que la "Correlación elemento-total corregida, es el coeficiente de homogeneidad corregido. Si es cero o negativo se elimina" (p. 7).

Como el valor obtenido no está en ninguno de los casos mencionados y comparado con el valor reportado por Román, Pérez y Moreno (2016) que indican que las validaciones de contenido ya han sido informadas, como la fiabilidad es de $\alpha \approx 0,80$, entonces se trabajó con un instrumento de un valor alto de fiabilidad.

\section{RESULTADOS}

En la figura 2 se muestran los porcentajes de aciertos que alcanzan por pregunta los estudiantes donde el $3,274 \%$ no aciertan, aciertan un $25 \%$ de preguntas el $7,44 \%$, aciertan en el $75 \%$ de preguntas $36 \%$ y alcanzan el $100 \%$ de respuestas correctas el $32,14 \%$. La mayor parte de los estudiantes logra resolver de 2 a 4 preguntas por lo que se puede considerar que en ellos existe un dominio en esta habilidad algorítmica del pensamiento computacional.

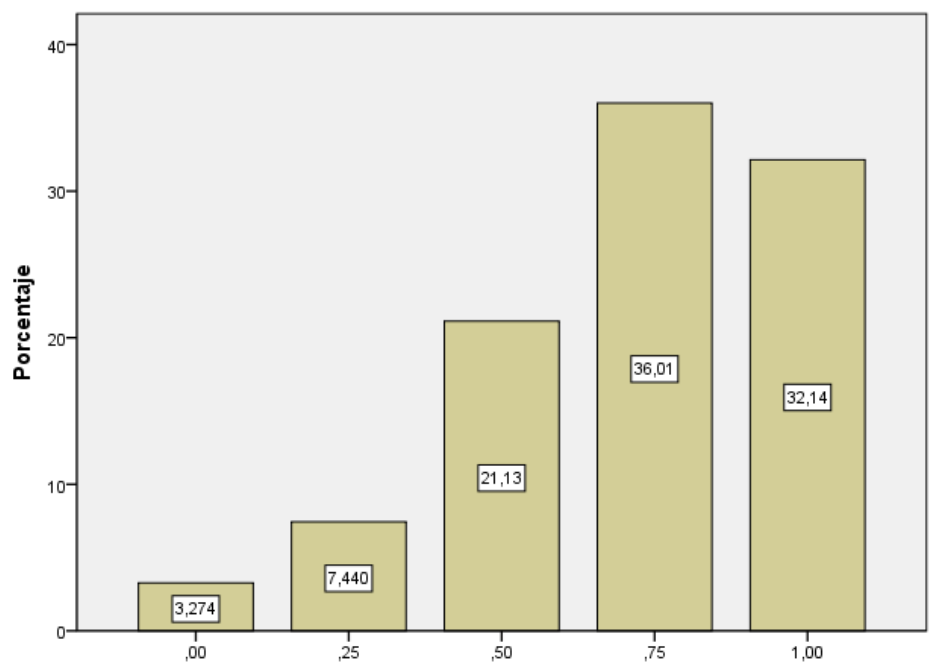

Figura 2. Promedios de respuestas problemas sobre algoritmos de orientación espacial. Fuente: Elaboración propia. 
En la figura 3 se observa que no tienen un solo acierto el 1,19\% de los estudiantes mientras el $3,869 \%$ alcanza un $13 \%$, el $6,25 \%$ de los estudiantes alcanzan un $25 \%$ de respuestas acertadas, el $13,69 \%$ alcanza un $38 \%$ de aciertos, el $19,35 \%$ resuelve correctamente el $50 \%$ de los problemas, el $22,92 \%$ resuelve el $63 \%$ acertadamente, el $23,81 \%$ responde correctamente el $75 \%$ de las preguntas, $8,036 \%$ de los estudiantes resuelven correctamente el $88 \%$ de los problemas y finalmente el $0,893 \%$ de los participantes resuelve correctamente el $100 \%$. La mayoría de los participantes se ubican en un rango de resolver correctamente el $38 \%$ al $75 \%$ los problemas referidos al empleo de bucles.

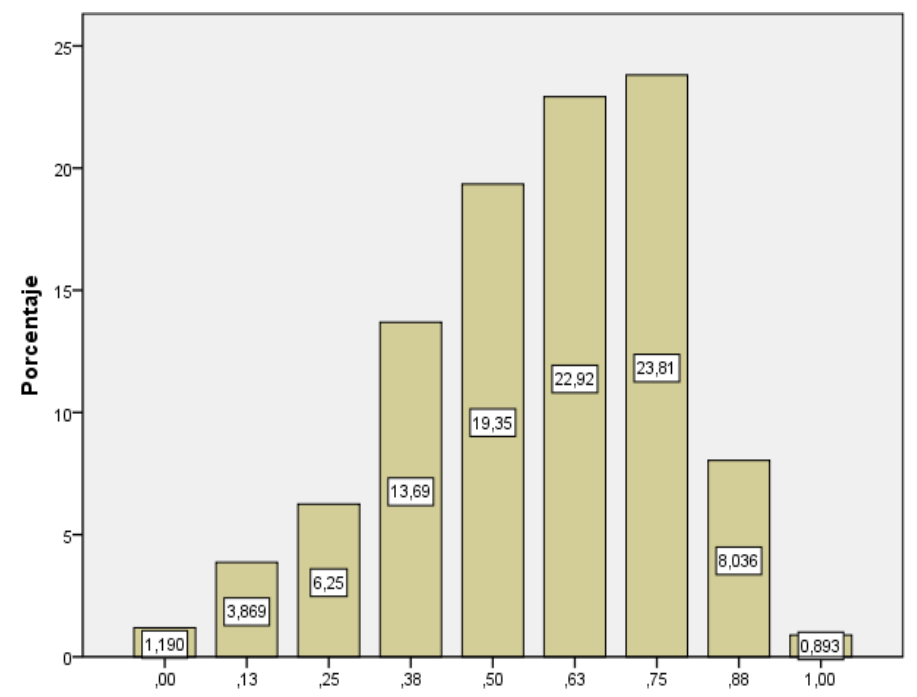

Figura 2. Promedios de respuestas problemas sobre bucles. Fuente: Elaboración propia.

Se puede considerar que obtienen un nivel medio de aciertos.

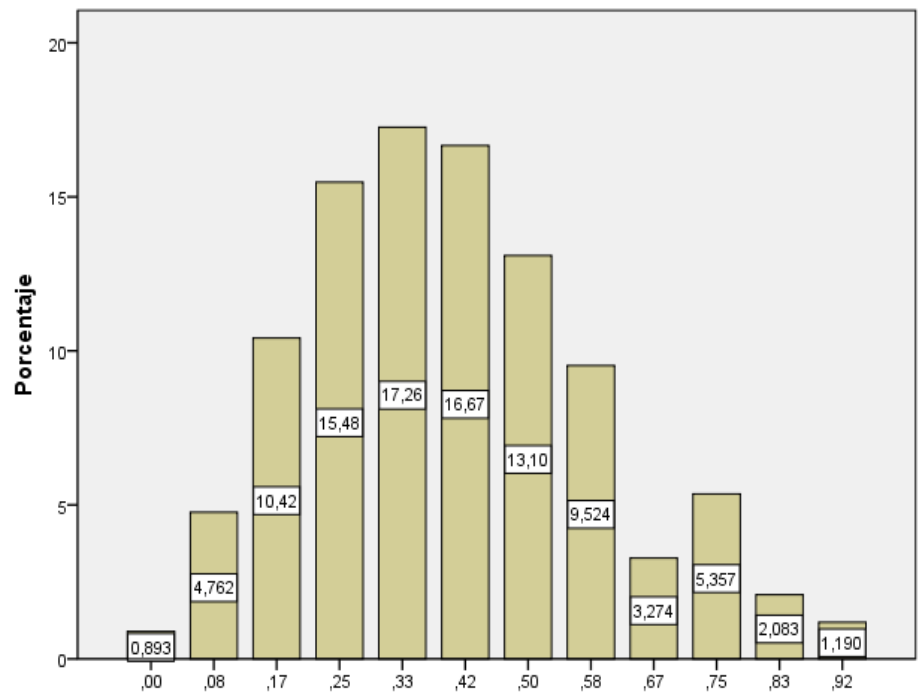

Figura 4. Promedios de respuestas problemas sobre toma de decisiones. Fuente: Elaboración propia.

La figura 4 muestra los resultados alcanzados por los estudiantes al resolver problemas sobre condiciones en donde el $0,893 \%$ posee un porcentaje de 0 aciertos, $4,762 \%$ alcanza un $8 \%$ de aciertos, el $10,42 \%$ logra $17 \%$ de aciertos, el $15,48 \%$ de los estudiantes logra resolver correctamente el $25 \%$ de los problemas, $17,26 \%$ resuelve el $33 \%$ de los problemas, $16,67 \%$ de los estudiantes resuelve el $42 \%$ de los problemas, $13,10 \%$ alcanza a resolver correctamente el $50 \%$ de los problemas, un porcentaje de $3,724 \%$ pudo resolver el $67 \%$ de los problemas, el $5,357 \%$ obtuvo el $75 \%$ de los problemas resueltos correctamente, el 2,083\% logró resolver 
correctamente el $83 \%$ de los problemas y el 1,190\% alcanzó a resolver el $92 \%$ de los problemas correctamente. Se puede considerar que obtienen un nivel bajo de aciertos en las respuestas.

En la figura 5 se puede observar los porcentajes de aciertos en las preguntas relacionadas a la elaboración de subrutinas el $5,655 \%$ de los estudiantes tienen $0 \%$ de aciertos, el $16,07 \%$ alcanza el $13 \%$ de aciertos, el $21,13 \%$ obtuvo $25 \%$ de aciertos, el $22,62 \%$ logra el $38 \%$ de aciertos, el $14,88 \%$ obtiene el $50 \%$ de aciertos, el $11,31 \%$ logra $63 \%$ de aciertos, el $4,762 \%$ obtuvo $75 \%$ de respuestas acertadas, un $2,976 \%$ de los estudiantes alcanza el $88 \%$ de aciertos y finalmente el $0,595 \%$ obtuvo $100 \%$ de aciertos.

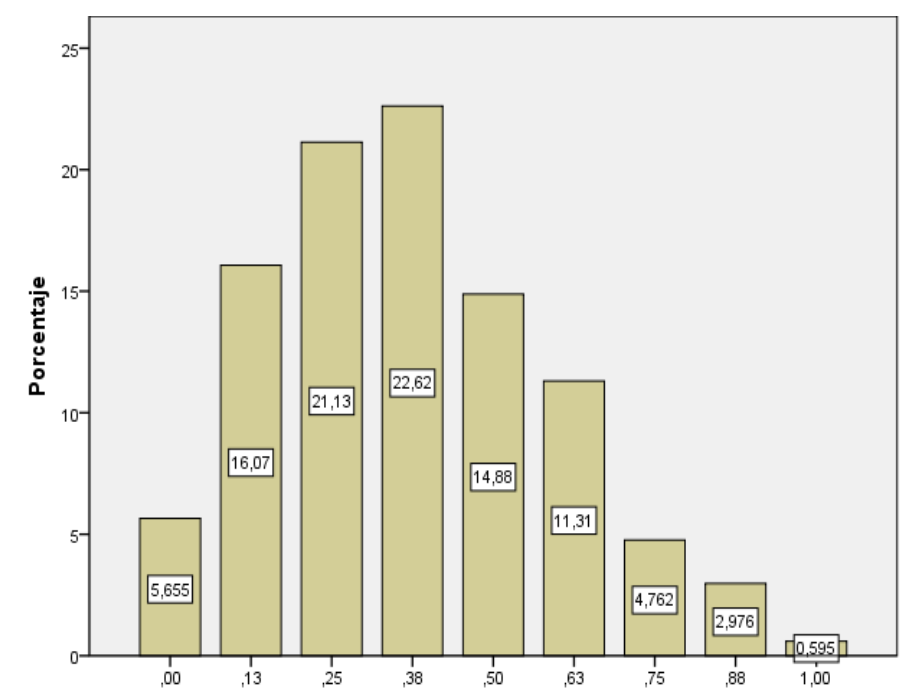

Figura 5. Promedios de respuestas problemas con subrutinas. Fuente: Elaboración propia.

La mayoría de los participantes se ubica en un rango de resolver correctamente entre $25 \%$ al $50 \%$ en los problemas referidos al empleo de condiciones. Se puede considerar un nivel bajo de aciertos en las respuestas que puede guardan relación con el escaso desarrollo del análisis frente a los problemas.

\subsection{Correlaciones}

Con el interés de establecer el grado de relación existente entre las habilidades seleccionadas en la investigación, se aplicó a los datos el coeficiente de correlación de Pearson a los datos obtenidos que pretenden responder a tres cuestiones que son:

\subsection{1. ¿Existe relación entre orientación espacial y la toma de decisiones en la resolución} de problemas?

Tabla 2. Correlaciones

\begin{tabular}{|c|c|c|c|}
\hline & & $\begin{array}{l}\text { Promedio de } \\
\text { direcciones }\end{array}$ & $\begin{array}{l}\text { Promedio de } \\
\text { condicionales }\end{array}$ \\
\hline \multirow[t]{4}{*}{$\begin{array}{l}\text { Promedio de orientación } \\
\text { espacial }\end{array}$} & $\begin{array}{l}\text { Correlación de Pearson } \\
\text { Sig. (bilateral) }\end{array}$ & 1 & $\begin{array}{l}, 421^{\star *} \\
, 000\end{array}$ \\
\hline & $\begin{array}{l}\text { Suma de cuadrados y productos } \\
\text { vectoriales }\end{array}$ & 23,231 & 7,122 \\
\hline & Covarianza & ,069 & ,021 \\
\hline & $\mathrm{N}$ & 336 & 336 \\
\hline \multirow[t]{4}{*}{$\begin{array}{l}\text { Promedio de toma de } \\
\text { decisiones }\end{array}$} & $\begin{array}{l}\text { Correlación de Pearson } \\
\text { Sig. (bilateral) }\end{array}$ & $\begin{array}{l}, 421^{\star *} \\
, 000\end{array}$ & 1 \\
\hline & $\begin{array}{l}\text { Suma de cuadrados y productos } \\
\text { vectoriales }\end{array}$ & 7,122 & 12,317 \\
\hline & Covarianza & ,021 & ,037 \\
\hline & $\mathrm{N}$ & 336 & 336 \\
\hline
\end{tabular}


Una vez realizado el estudio correlacional (coeficiente de Pearson) entre orientación espacial y toma de decisiones, se observa que hay una relación directa significativa de 0,421 entre ambas por lo que se asume que existe influencia entre las dos habilidades del pensamiento computacional.

\subsection{2. ¿Existe relación entre la toma de decisiones y el empleo de bucles de repetición en} la resolución de problemas?

Tabla 3. Correlaciones

\begin{tabular}{|ll|r|r|}
\hline & & $\begin{array}{c}\text { Promedio de } \\
\text { condicionales }\end{array}$ & \multicolumn{1}{c|}{$\begin{array}{c}\text { Promedio de } \\
\text { bucles }\end{array}$} \\
\hline Promedio de condicionales & Correlación de Pearson & 1 &, $349^{* *}$ \\
& Sig. (bilateral) &, 000 \\
& Suma de cuadrados y & & 4,585 \\
& productos vectoriales & 12,317 &, 014 \\
& Covarianza &, 037 & 336 \\
& $\mathrm{~N}$ & 336 & 1 \\
& Correlación de Pearson &, $349^{* *}$ &, 000 \\
& Sig. (bilateral) & 4,585 & 13,983 \\
& Suma de cuadrados y &, 014 &, 042 \\
& productos vectoriales & 336 & 336 \\
\hline
\end{tabular}

Fuente: Cuestionario aplicado a estudiantes. Elaboración: Investigadores

Una vez realizado el estudio correlacional (coeficiente de Pearson) toma de decisiones y empleo de bucles de repetición en la resolución de problemas, se observa que hay una relación directa significativa de 0,349 entre ambas por lo asume que existe influencia entre las dos habilidades del pensamiento computacional.

\subsection{3. ¿Existe relación entre toma de decisiones y el empleo de funciones o subrutinas en} la resolución de problemas?

Tabla 4. Correlaciones

\begin{tabular}{|c|c|c|c|}
\hline & & $\begin{array}{l}\text { Promedio de } \\
\text { funciones y } \\
\text { subrutinas }\end{array}$ & $\begin{array}{l}\text { Promedio de } \\
\text { condicionales }\end{array}$ \\
\hline \multirow{5}{*}{$\begin{array}{l}\text { Promedio de funciones } y \\
\text { subrutinas }\end{array}$} & Correlación de Pearson & 1 & ,390* \\
\hline & Sig. (bilateral) & & ,000 \\
\hline & $\begin{array}{l}\text { Suma de cuadrados y } \\
\text { productos vectoriales }\end{array}$ & 15,837 & 5,452 \\
\hline & Covarianza & 047 & ,016 \\
\hline & N & 336 & 336 \\
\hline \multirow[t]{2}{*}{ Promedio de condicionales } & Correlación de Pearson & ,390* & 1 \\
\hline & Sig. (bilateral) &, 000 & \\
\hline
\end{tabular}




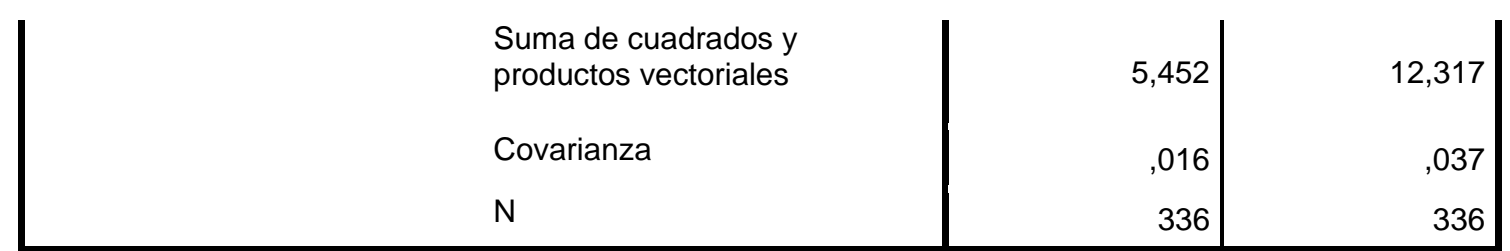

Fuente: Cuestionario aplicado a estudiantes. Elaboración: Investigadores

Una vez realizado el estudio correlacional (coeficiente de Pearson) entre toma de decisiones y empleo de funciones o subrutinas, se observa que hay una relación directa significativa de 0,390 entre ambas por lo que existe influencia entre las dos habilidades del pensamiento computacional.

\section{DISCUSIÓN Y CONCLUSIONES}

Entre los principales hallazgos de la investigación, se observa que los resultados alcanzados por los estudiantes son bastante heterogéneos, difieren de acuerdo con la habilidad evaluada mediante el cuestionario, siendo los relacionados con algoritmos de orientación espacial y empleo de bucles los que alcanzan altos niveles de acierto entre los estudiantes. Mientras que en problemas sobre toma de decisiones y uso de subrutinas muestran niveles elevados de dificultad y pocos aciertos.

Se observa que existe relación entre el desarrollo que tengan en la habilidad de toma decisiones y otras habilidades como empleo de bucles de repetición o elaboración de subrutinas, que se expresa en la dificultad de los estudiantes a tomar una decisión razonada y lógica que permita resolver un problema.

En resumen, la población investigada no muestra solvencia en las habilidades investigadas de manera que pueda resolver problemas apoyados en el uso de la tecnología y el razonamiento lógico.

Se comparó los resultados con la investigación en el grupo de estudiantes del primer semestre de la Carrera de Informática de la Universidad Central del Ecuador, tomando en cuenta características similares: edad y nivel de formación, que realizó Pérez (2017) sobre el nivel de desarrollo del pensamiento computacional empleando la herramienta Scratch.

Los resultados de esa investigación contrastados con los obtenidos en la presente, muestran coincidencias en la dificultad al resolver problemas de tipo algorítmico, tanto en los estudiantes de la Universidad Central del Ecuador, que obtuvo en el uso de instrucciones "un $37 \%$ de aciertos frente al 63\% que comete errores" (Pérez, 2017, p. 301), así como en el Instituto Tecnológico Superior Sucre, donde se observó que los estudiantes muestran altos niveles de dificultad frente a problemas que emplean toma de decisiones y sentencias de repetición.

En el aspecto del empleo de subrutinas, que se evidencia en la práctica mediante la capacidad de dividir un problema en partes para su solución, los resultados presentan escasos niveles de eficiencia que pueden incidir en su profesional. La inclusión del PC puede aportar en ese sentido tomando en cuenta lo que manifiesta Hemmendinger (2010):

El objetivo no es enseñar a pensar a todo el mundo como un informático, sino como médicos, arquitectos o abogados, etc. que comprendan cómo usar la computación para solucionar los problemas de sus profesiones y disciplinas y formular nuevas cuestiones que puedan ser exploradas de modo fructífero con dichas herramientas conceptuales. (p.4)

Se considerar la probabilidad que los estudiantes del nivel educativo superior no han desarrollado los elementos del pensamiento computacional que aportan a la resolución de problemas de manera eficiente.

En cuanto a las limitaciones de la investigación se podría considerar que el instrumento no considera elementos del pensamiento computacional como creatividad, reuso entre otros y que podrían aportar mayores elementos para establecer el desarrollo que alcanzan los estudiantes, 
por lo que sería conveniente continuar con la investigación aplicando otras metodologías que informen sobre otros aspectos no investigados.

También, existe la limitación que corresponde al estudio en una institución, lo que particulariza los resultados. Es importante replicar la experiencia en otras instituciones educativas de nivel tecnológico, de manera que oriente la toma de decisiones sobre la presencia - no una la asignatura que desarrolle competencias informáticas relacionadas con el pensamiento computacional, en la formación de los profesionales tecnológicos en el país.

Como consideración final se puede pensar en investigaciones a futuro que amplíen la visión a nivel nacional acerca del desarrollo del pensamiento computacional, en los estudiantes que ingresan a las instituciones de educación superior, toda vez que en el bachillerato y educación básica ecuatoriana no existe una asignatura que aporte en su desarrollo, así como son escasas las investigaciones acerca del tema en el país.

\section{ENLACES}

Ubicación del instrumento:

https://drive.google.com/drive/folders/OB7vOwLMkIOOzdWFYTF9rZzZHRIE

\section{REFERENCIAS BIBLIOGRÁFICAS}

CSTA y ISTE (2011). Pensamiento computacional. Caja de herramientas para líderes. Recuperado de http://eduteka.icesi.edu.co/pdfdir/PensamientoComputacional1.pdf

García-Bellido, R., González, J. y Jornet M. (2015). Introducción al SPSS. Universidad de Valencia. Grupo de innovación educativa. Recuperado de https://www.uv.es/innomide/spss/SPSS/SPSS_0801B.pd

Hitschfeld, N., Pérez, J. y Simmonds, J. (2015). Pensamiento computacional y programación a nivel escolar en Chile: El valor de formar a los innovadores tecnológicos del futuro. Bits de Ciencia, 12, 28-33.

Hemmendinger, D. (2010). Aplea for modesty. ACM Inroads, 1(2), 4-7. doi: https://doi. org/10.1145/1805724.1805725

Kahneman, D. (2011). Pensar rápido, pensar despacio. Recuperado de https://catedradatos.com.ar/media/kahneman_pensar.pdf

Llopis F., Corbí A., Llorens, F. (2001). Fundamentos de programación. Vol. I. Metodología. Recuperado de http://bvirtual.uce.edu.ec:2057/a/663/fundamentos-de-programacion.-vol.i.-metodologia

Ministerio de Educación del Ecuador. (2017). Agenda Educativa Digital. Enfoque de la Agenda educativa digital. Quito: Ed. Ministerio de Educación. Recuperdado de www.educacion.gob.ec

Pérez, H. (2017). Uso de Scratch como herramienta para el desarrollo del pensamiento computacional en programación y de la carrera de Informática de la Universidad Central del Ecuador. Tesis doctoral Universidad de Alicante. Recuperado de: https://dialnet.unirioja.es/servlet/tesis?codigo $=121416$

Román, M., Pérez, J., y Moreno, J. (2016). Does computational thinking correlate with personality? The non-cognitive side of computational thinking. Retrieved from: https://dl.acm.org/citation.cfm?id=3012496

Royal Society Education. (2012). Shut down or restart. The way foward for computing of UK schools. London. Retrieved from https://royalsociety.org/ /media/education/computing-inschools/2012-01-12-computing-in-schools.pdf 
Suárez, J., Maiz, F. y Meza, M. (2010). Inteligencias múltiples: una innovación pedagógica para potenciar el proceso enseñanza aprendizaje. Revista Revinpost. Retrieved from: https://www.redalyc.org/pdf/658/65822264005.pdf

Scott, C.L. 2015. El futuro del aprendizaje 2 ¿Qué tipo de aprendizaje se necesita en el siglo XXI? Investigación y Prospectiva en Educación. Documentos de Trabajo ERF, No. 14. París: UNESCO.

Wing, J. (2006). Computational thinking. COMMUNICATIONS OF THE ACM, 49(3), p. 33-35. Retrieved from https://www.cs.cmu.edu/ 15110-s13/Wing06-ct.pdf

Wing, J. (2008). Computational thinking and thinking about computing. Philosophical Transactions of Royal Society, 366, 3717-3725. https://doi.org/10.1098/rsta.2008.0118 Wing08a.pdf

\section{INFORMACIÓN SOBRE LOS AUTORES}

\section{Hamilton Omar Pérez Narváez \\ Universidad Central del Ecuador}

Licenciado en Ciencias de la Educación especialización Informática, Especialista en Entornos Virtuales OEl. Magíster en Educación Superior y Doctor en Investigación Educativa en la Universidad de Alicante. Ha escrito varios artículos relacionados con la Tecnología Educativa y el pensamiento computacional. Profesor de Bachillerato Técnico en Informática, Profesor de la Universidad Central del Ecuador en las cátedras de: Programación, Didáctica de la Informática y Pedagogía, profesor de la Pontificia Universidad Católica del Ecuador en cursos de TIC.

Ha sido tutor de tesis de grado y posgrado en la Facultad de Filosofía, coordinador de Maestría en Educación mención Gestión del aprendizaje mediado por TIC. Actualmente es Director de la carrera de Pedagogía de las Ciencias Experimentales en Informática y coordinador de la Red ecuatoriana de tecnología e innovación educativa.

\section{Alex Álvarez-Zurita \\ Universidad Central del Ecuador}

Obtuvo su título Magíster en Tecnología Educativa y Competencias Digitales en la Universidad Internacional de la Rioja (UNIR) España en 2019. Obtuvo el título de Magíster en Educación Superior en la Facultad de Filosofía, Letras de Ciencias de la Educación de la Universidad Central del Ecuador (Ecuador) en 2014 y obtuvo el título de Licenciado en Ciencias de la Educación Mención Informática en la Facultad de Filosofía, Letras de Ciencias de la Educación de la Universidad Central del Ecuador (Ecuador) en 2008. Actualmente es profesor de la Facultad de Filosofía, Letras y Ciencias de la Educación de la Universidad Central Ecuador. Y profesor tiempo completo en el Instituto Tecnológico Superior Sucre.

\section{Carlos Roberto Guevara Herrera}

Profesor Instituto Tecnológico Superior "Sucre"

Obtuvo su título Magíster en Tecnología Educativa y Competencias Digitales en la Universidad Internacional de la Rioja (UNIR) España en 2019. Obtuvo el título de Licenciado en Ciencias de la Educación Mención Informática en la Facultad de Filosofía, Letras de Ciencias de la Educación de la Universidad Central del Ecuador (Ecuador) en 2008. Actualmente es profesor del Colegio María de Nazaret y profesor en el Instituto Tecnológico Superior Sucre.

Los textos publicados en esta revista están sujetos a una licencia de Reconocimiento 4.0 España de Creative Commons. Puede copiarlos, distribuirlos, comunicarlos públicamente y hacer obras derivadas siempre que reconozca los créditos de las obras (autoría, nombre de la revista, institución editora) de la manera especificada por los autores o por la revista. La licencia completa se puede consultar en:Licencia Creative Commons Atribución-NoComercial-Compartir por iqual 4.0 Internacional. 